\title{
Respective Contribution of Chronic Conditions to Disability in France: Results from the National Disability- Health Survey
}

\author{
Clémence Palazzo ${ }^{1,2,3,4 *}$, Jean-François Ravaud ${ }^{5,6,7}$, Ludovic Trinquart ${ }^{1,2}$, Marie Dalichampt ${ }^{1,2}$, \\ Philippe Ravaud ${ }^{1,2,3}$, Serge Poiraudeau ${ }^{3,4,5}$
}

1 U738, INSERM, Paris, France, 2 Centre d'Épidémiologie Clinique, Hôpital Hôtel Dieu AP-HP, Paris, France, 3 Université Paris Descartes, PRES, Paris, France, 4 Service de rééducation et réadaptation de l'appareil locomoteur et des pathologies du rachis, Hôpital Cochin AP-HP, Paris, France, $\mathbf{5}$ Institut fédératif de recherche sur le handicap, INSERM, Paris, France, 6 U988, INSERM, Villejuif, France, 7 UMR 8211, CNRS, Villejuif, France

\begin{abstract}
Background: Representative national data on disability are becoming increasingly important in helping policymakers decide on public health strategies. We assessed the respective contribution of chronic health conditions to disability for three age groups (18-40, 40-65, and >65 years old) using data from the 2008-2009 Disability-Health Survey in France.

Methods: Data on 12 chronic conditions and on disability for 24,682 adults living in households were extracted from the Disability-Health Survey results. A weighting factor was applied to obtain representative estimates for the French population. Disability was defined as at least one restriction in activities of daily living (ADL), severe disability as the inability to perform at least one ADL alone, and self-reported disability as a general feeling of being disabled. To account for comorbidities, we assessed the contribution of each chronic disorder to disability by using the average attributable fraction (AAF).

Findings: We estimated that 38.8 million people in France $(81.7 \%$ [95\% Cl $80.9 ; 82.6])$ had a chronic condition: $14.3 \%$ $(14.0 ; 14.6)$ considered themselves disabled, $4.6 \%(4.4 ; 4.9)$ were restricted in ADL and $1.7 \%(1.5 ; 1.8)$ were severely disabled. Musculoskeletal and sensorial impairments contributed the most to self-reported disability (AAF $15.4 \%$ and $12.3 \%$ ). Neurological and musculoskeletal diseases had the largest impact on disability (AAF $17.4 \%$ and $16.4 \%$, respectively). Neurological disorders contributed the most to severe disability (AAF 31.0\%). Psychiatric diseases contributed the most to disability categories for patients $18-40$ years old (AAFs $23.8 \%-40.3 \%$ ). Cardiovascular conditions were also among the top four contributors to disability categories (AAFs $8.5 \%-11.1 \%$ ).
\end{abstract}

Conclusions: Neurological, musculoskeletal, and cardiovascular chronic disorders mainly contribute to disability in France. Psychiatric impairments have a heavy burden for people 18-40 years old. These findings should help policymakers define priorities for health-service delivery in France and perhaps other developed countries.

Citation: Palazzo C, Ravaud J-F, Trinquart L, Dalichampt M, Ravaud P, et al. (2012) Respective Contribution of Chronic Conditions to Disability in France: Results from the National Disability-Health Survey. PLoS ONE 7(9): e44994. doi:10.1371/journal.pone.0044994

Editor: Deborah Kado, UCSD School of Medicine, United States of America

Received January 25, 2012; Accepted August 15, 2012; Published September 14, 2012

Copyright: (c) 2012 Palazzo et al. This is an open-access article distributed under the terms of the Creative Commons Attribution License, which permits unrestricted use, distribution, and reproduction in any medium, provided the original author and source are credited.

Funding: This work was supported by the French National Institute of Statistics and Economic Studies (INSEE) and the French Head Office of Research, Studies, Evaluation and Statistics (DREES) of the Social Affairs Ministry (contract PA 11/R 10100LL). The French National Institute of Statistics and Economic Studies (INSEE) and the French Head Office of Research, Studies, Evaluation and Statistics (DREES) of the Social Affairs Ministry had no role in study design, data collection and analysis, decision to publish, or preparation of the manuscript. The authors received a grant from Pfizer France, which had no role in study design, data collection and analysis, decision to publish, or preparation of the manuscript.

Competing Interests: The authors have read the journal's policy and have the following conflict: S. Poiraudeau received fees from Pfizer for consultancy. This does not alter the authors' adherence to all the PLoS ONE policies on sharing data and materials. The other authors have declared that no competing interests exist. There is no patent, product in development or marketed product,... etc., associated with Pfizer.

* E-mail: clemence.palazzo@cch.aphp.fr

\section{Introduction}

Disability is fast becoming a concern because of its increasing prevalence owing to the aging of the population, the increased risk of disability in older people, and the global increase in chronic conditions [1]. According to the 2004 Global Burden of Disease estimates [2], chronic non-communicable diseases contributed to $68 \%$ of 751 million years lived with disability worldwide and were largely depression, sensorial impairments and osteoarthritis in elderly people.
Preferences in public health priorities differ when the target is mortality or disability. Studies of the contribution of different diseases to mortality have been numerous, but those investigating the contribution of disability are lacking. Several authors compared the contribution of different disorders to disability $[3,4,5,6]$ to help policymakers decide on intervention and preventive strategies. However, the studies vary in terms of groups studied, diseases and approaches used to define and measure disability. Moreover, these data often concern co-morbid situations, so a reliable estimate of the respective weight of diseases in 
global disability is difficult. This situation is particularly true for studies in developed countries and involving elderly people.

Use of the average attributable fraction (AAF) [7] has been recently suggested to solve the problem of the respective weight of diseases to global disability [8]. Briefly, the AAF is the expected proportion of cases of disability preventable by the additional elimination of the condition of interest after a random collection of other disorders has been eliminated [7].

In 2008-2009, the national representative Disability-Health Survey was carried out to describe disabilities in the population in France. We used the AAF to analyze data from this survey to assess the current contribution of chronic conditions to disability.

\section{Methods}

\section{Ethics}

This study was planned as a research project. It was performed in collaboration with the French National Institute of Statistics. This study was declared of public interest by the CNIS (Conseil National d'Information Statistique) and was approved by the CNIL (Commission Nationale de l'Informatique et des Libertés, French law no. 78-17). According to the French law, written informed consent was not required for this type of study.

\section{Disability-Health Survey design}

The Disability-Health Survey (available at http://www.sante. gouv.fr/handicap-sante.html) was a national cross-sectional survey with a two-stage design (Figure 1) aiming to describe disability and handicap in France. It was developed by the French National Institute of Statistics and Economic Studies (INSEE) and the French Head Office of Research, Studies, Evaluation and Statistics of the Social Affairs Ministry.

First, a preliminary filter-survey was performed to identify and stratify disabilities. Briefly, a questionnaire was sent to 141138 representative households from the 2006 census in France and the INSEE survey districts. The response rate was $79.1 \%$. The questionnaire has 26 sections on health, activity limitations, help, administrative recognition of disability, and personal perception of the situation. According to their answers, people were classified into four levels of presumed disability severity, from 1 (no disability) to 4 (high level of disability). The survey also involved intensive sampling in several geographic areas to obtain representative data in these areas. Stratification into 44 strata was based on the four presumed disability levels and the 11 geographic areas of residence.

For sample selection, randomisation involved a high sampling rate for the most severely disabled group and a low sampling rate for people without daily living restrictions (the largest group). Each of the resulting groups was allocated a specific sampling coefficient that increased with the probability or severity of the presumed handicap. The sampling rate was also higher for people living in the geographic areas that were more intensively sampled. Data were collected from March to July 2008, including 39065 individuals across the territory departments in France. Trained investigators used the computer-assisted interview (CAPI) format to collect data from people in their homes. A household member or a proxy could answer for identified survey respondents not able to answer alone. The response rate was $76.6 \%$, corresponding to 29931 subjects (24 682 adults older than 18 years) with complete data.

Each respondent was assigned a weight reflecting the probability of being investigated (depending on presumed disability severity and geographic area of residence) and answering the question- naire, which allowed for estimating representative results at a national level.

\section{Measurement of disability}

We considered three situations of disability on the basis of subjects' self-reports.

We defined overall disability and severe disability according to Katz' activities of daily living (ADL) score [9], which includes six items: bathing, dressing, toileting, transferring (moving from bed and chair), preparing a meal and self-feeding. Respondents were asked about the degree of difficulty in performing these activities on a scale from 0 (no difficulty) to 4 (inability to do it alone). Disability was defined as any restriction in performing ADL with or without technical aid [10] and severe disability as the inability to perform at least one ADL alone [11]. Self-reported disability was considered a positive answer to the question "Do you consider that you have a disability?"

\section{Assessment of chronic conditions and socio- demographic characteristics}

Chronic diseases were self-reported. Interviewers presented a checklist of 52 disorders classified in 12 groups according to the $10^{\text {th }}$ International Classification of Diseases criteria [12]: cardiovascular, musculoskeletal, neurological, psychiatric, respiratory, dermatological, endocrine, urological, digestive, and sensorial diseases; cancer; and sequelae of injury (Table 1).

The diagnosis of condition had to be from a physician, except for symptoms such as back pain, neck pain and headache. We also assumed disorders not treated during the previous year and stroke without any sequelae as "no disease."

The survey collected data on sex, age, marital status, educational attainment, working status, occupation class, place of residence (urban/rural), and living situation (alone or not).

\section{Statistical analysis}

The final weighting factors combined design weights and nonresponse weights. Design weights were the inverse of the sampling fraction, depending on presumed disability severity and geographic area of residence. Probability of non-response was estimated by logistic regression, with age, gender, type of household, marital status, and nine questions about health and disability used as independent variables. Finally, calibration was based on geographic area of residence, age and gender.

For the descriptive analysis, we reported the prevalence of diseases, summarized socio-demographic characteristics and described disabilities by frequencies, means and their $95 \%$ confidence intervals $(95 \% \mathrm{CIs})$ corrected with the sampling weight.

To assess the respective contribution of chronic diseases to disability, we used three steps. First, we calculated the gross attributable fraction (AF) of each chronic disorder to disability, defined as the fraction of the overall rate of disability that could be avoided if that disease was eliminated in the population [13]. AFs were calculated using Levin's formula [13]:

$$
\mathrm{AF}=\mathrm{Pe} .(\mathrm{RR}-1) /(1+\text { Pe. }(\mathrm{RR}-1))
$$

Pe is the prevalence of the disease and $R R$ the relative risk estimate to be disabled when having the disease. The $95 \%$ confidence intervals were calculated with Fleiss' formula [14]. The main limitation of $\mathrm{AF}$ is that it measures not the single contribution of the disease of interest. Considering an example from our dataset, an AF of $20 \%$ for musculoskeletal impairment means that for $20 \%$ of people reporting disability, the disability 


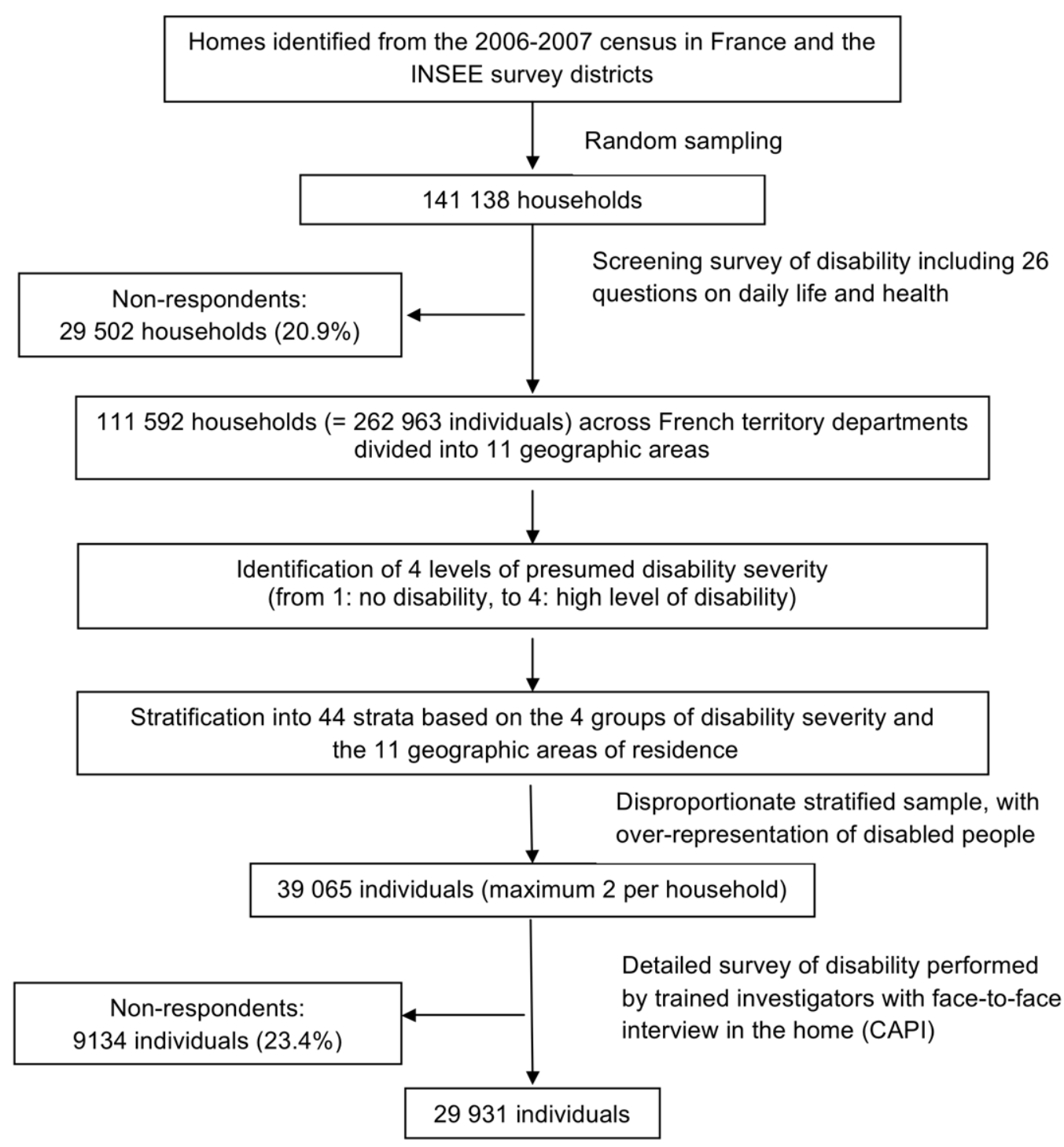

Figure 1. Design of the representative national "Disability-Health" survey. INSEE = French National Institute of Statistics and Economic Studies.

doi:10.1371/journal.pone.0044994.g001

can be globally attributed to musculoskeletal disorders; that is to musculoskeletal disorders alone, but also to musculoskeletal disorders associated with other chronic disease(s), such as neurological or psychiatric conditions. Consequently, AF can overestimate the potential impact of musculoskeletal disorders [8], and the sum of the AFs of all chronic conditions can add up to more than $100 \%$, which can produce some confusion when interpreting the results. We grouped diseases with a low AF (arbitrarily $\leq 15 \%$; "other diseases") for the following calculations.

Next, we calculated the AAF [7], considered to be a relevant methodology for use in co-morbid situations, and defined as the expected proportion of disability preventable by the additional elimination of the chronic condition of interest, after adjustment for a random collection of other disorders [7]. Briefly, the AAF is based on the idea of partitioning disability into a set of risk factors, including chronic disorders. For the previous example, an AAF of $20 \%$ for musculoskeletal disorders means that for $20 \%$ of people reporting disability, the disability can be attributed only to musculoskeletal diseases. Contrary to AF, the AAF of a chronic disorder reflects its single contribution to disability, and the sum of the AAFs of all chronic conditions should not add up to more than $100 \%$. The method of calculating the AAF is explained in detail with an example in Appendix S1. To our knowledge, no method exists for calculating AAF confidence intervals.

Finally, AAFs were multiplied by the total number of disabled subjects to estimate the expected number of disabled subjects in whom disability would be prevented by eliminating each chronic disorder.

Because prevalence rates of diseases and frequencies of disability vary by age, we analyzed these categories and AAFs in the overall population by three age groups: $18-40,40-65$, and $>65$ years. We adjusted for potential confounders identified a priori from the literature: sex [15], marital status [16], living situation (alone or not) [17], educational level [18] and place of residence (rural area, urban area $<200000$ people and urban area $\geq 200000$ people) [19].

Statistical analyses involved use of SAS 9.2 (SAS Inst, Cary, $\mathrm{NC}$ ). Sampling weights were taken into account with specific SAS 
Table 1. Chronic conditions and the included diseases evaluated in estimating the contribution of diseases to disability in the population in France.

\begin{tabular}{|c|c|}
\hline Chronic conditions & Including diseases \\
\hline Cancer & cancer (including lymphoid, haematopoietic and related tissue) \\
\hline Cardiovascular & $\begin{array}{l}\text { myocardial infarction, angina, stroke, heart failure, lower limb arterial occlusive disease, venous insufficiency and high } \\
\text { blood pressure }\end{array}$ \\
\hline Dermatological & psoriasis, eczema and slough \\
\hline Digestive & ulcer, cirrhosis (and other liver diseases) and food allergies \\
\hline Endocrine & diabetes mellitus, disorders of the thyroid gland and obesity (body mass index $\geq 30 \mathrm{~kg} / \mathrm{m}^{2}$ ) \\
\hline Musculoskeletal & $\begin{array}{l}\text { back pain, neck pain, scoliosis, rheumatoid arthritis, other inflammatory arthritis, knee and hip osteoarthritis, other type } \\
\text { of osteoarthritis and osteoporosis }\end{array}$ \\
\hline Neurological & headache, epilepsy, dementia, Parkinson's disease, multiple sclerosis and other unspecified neurological problems \\
\hline Psychiatric & depression, anxiety, autism, schizophrenia, trisomy 21 and other unspecified psychiatric impairments \\
\hline Respiratory & asthma, chronic bronchitis and hay fever \\
\hline Sensorial & eyesight problems ${ }^{b}$ and hearing loss ${ }^{c}$ \\
\hline Sequelae of injury & sequelae of injury \\
\hline Urological & urinary incontinence, infection of the urinary tract, lithiasis and prostate adenoma \\
\hline
\end{tabular}

This classification followed the $10^{\text {th }}$ International Classification of Diseases [12]; ${ }^{\text {a }}$ Calculated from reported height and weight as weight/height ${ }^{2}$; ${ }^{\text {b }}$ Eyesight problems included cataract, strabismus and glaucoma reported in the checklist of the questionnaire and a positive answer to the question: "Do you have any eyesight problems?"; ' Hearing loss included a positive answer to the questions: "Are you wearing a hearing aid?" or "Do you have any hearing problems?"

doi:10.1371/journal.pone.0044994.t001

procedures for handling complex sample designs [20]. AAFs were computed with the macro developed by Rückinger et al [8] that we modified to take into account sample design and adjustment for variables.

\section{Results}

Prevalence rates and socio-demographic characteristics of people with chronic conditions in France

The prevalence rates and socio-demographic characteristics of survey subjects reporting chronic diseases are in Table 2 (in detail in Table S1).

The mean age of the population was 48.4 years old (95\% CI $48.1 ; 48.7$ ). In the overall household population (47524 123 people): $37.7 \%(36.7 ; 38.7)$ were 18 to 40 years old, $42.8 \%$ $(41.8 ; 43.8)$ were 40 to 65 years old and $19.5 \%(18.8 ; 20.1)$ were $>65$ years old. In the overall household population, an estimated 38.8 million people $81.7 \%$ (80.9;82.6) had chronic conditions. The most frequently reported conditions were sensorial and musculoskeletal impairments. These impairments were reported by $80.1 \%$ $(79.2 ; 81.0)$ and $35.2 \%(34.2 ; 36.1)$, respectively, of the population with chronic conditions and by $65.4 \%(64.5 ; 66.4)$ and $28.7 \%$ $(27.9 ; 29.5)$, respectively, of the overall adult population.

People with chronic conditions were older than those in the overall adult population (mean age 51.4 (51.0;51.8) vs. 48.4 $(48.1 ; 48.7)$ years). People $>65$ years old represented $23.2 \%$ $(22.5 ; 23.9)$ of people reporting chronic diseases and $19.5 \%$ $(18.9 ; 20.1)$ of the overall adult population. This proportion was high for people reporting cancer $(46.5 \%$ (41.1;51.9)), cardiovascular diseases $(46.8 \%(45.2 ; 48.5))$ and urological impairments $(47.6 \%(44.2 ; 51.0))$. The mean number of chronic diseases was high for urological $(4.1(4.0 ; 4.2))$ and digestive disorders $(4.2$ $(4.0 ; 4.3))$. Chronic conditions were less frequent for men, except for sequelae of injury. Almost half of the estimated 3 million people with psychiatric disorders were single $(44.9 \%$ (41.9;47.9)). People with psychiatric and neurological diseases had high unemployment rates $(6.9 \%(5.4 ; 8.3)$ and $7.0 \%(5.6 ; 8.5)$, respectively).

Frequencies of chronic conditions with disability in the overall adult population

In total, $12.0 \%(11.8 ; 12.3)$ of people (approximately 5.7 million people) considered that they had a disability, with sensorial and musculoskeletal disorders the main chronic diseases $(82.8 \%$ $(81.7 ; 83.9)$ and $56.3 \%(54.9 ; 57.7)$, respectively) (Table 3$)$. In all, $3.9 \%(3.7 ; 4.1)$ (approximately 1.8 million people) were restricted in at least one ADL, particularly bathing and dressing, with sensorial and musculoskeletal conditions the most frequently reported diseases $(85.2 \%(83.3 ; 87.2)$ and $63.3 \%(60.7 ; 65.9))$. Finally, $1.4 \%$ (1.3;1.5) (approximately 0.67 million people) reported severe disability, with sensorial and cardiovascular disorders the most frequently reported diseases $(82.6 \% \quad(79.0 ; 86.3)$ and $53.3 \%$ $(48.9 ; 57.6))$.

\section{Contribution of chronic conditions to disability}

The highest $\mathrm{AFs}$ were for sensorial impairments (49.7\% $(49.5 ; 50.0)$ to $57.3 \%(57.2 ; 57.5))$ (Table S2). Even though the prevalence of neurological $(10.1 \%)$ or psychiatric diseases $(6.3 \%)$ was not high, the AF findings for these diseases were significant because of the high relative risk of being disabled with these diseases. In contrast, respiratory disorders had a higher prevalence $(11.5 \%)$ but lower AFs $(<15.0 \%)$. We grouped conditions with $\mathrm{AFs}_{\mathrm{S}} \leq 15.0 \%$ in each age group: cancer and digestive, respiratory and dermatological diseases.

AAFs estimates for disability, severe disability and self-reported disability are in table 4,5 and 6, respectively, and AAFs are represented in Figure 2.

Neurological, musculoskeletal, and cardiovascular diseases had the greatest impact on disability (AAF 17.4\%, 320000 people; $16.4 \%, 290000$ people; and 11.1\%, 180000 people). The AAF for neurological conditions was high for people $>65$ years old (21.0\%, 240000 people), whereas that for psychiatric disorders was highest for people $\leq 40$ years old $(27.4 \%, 40000$ people). 


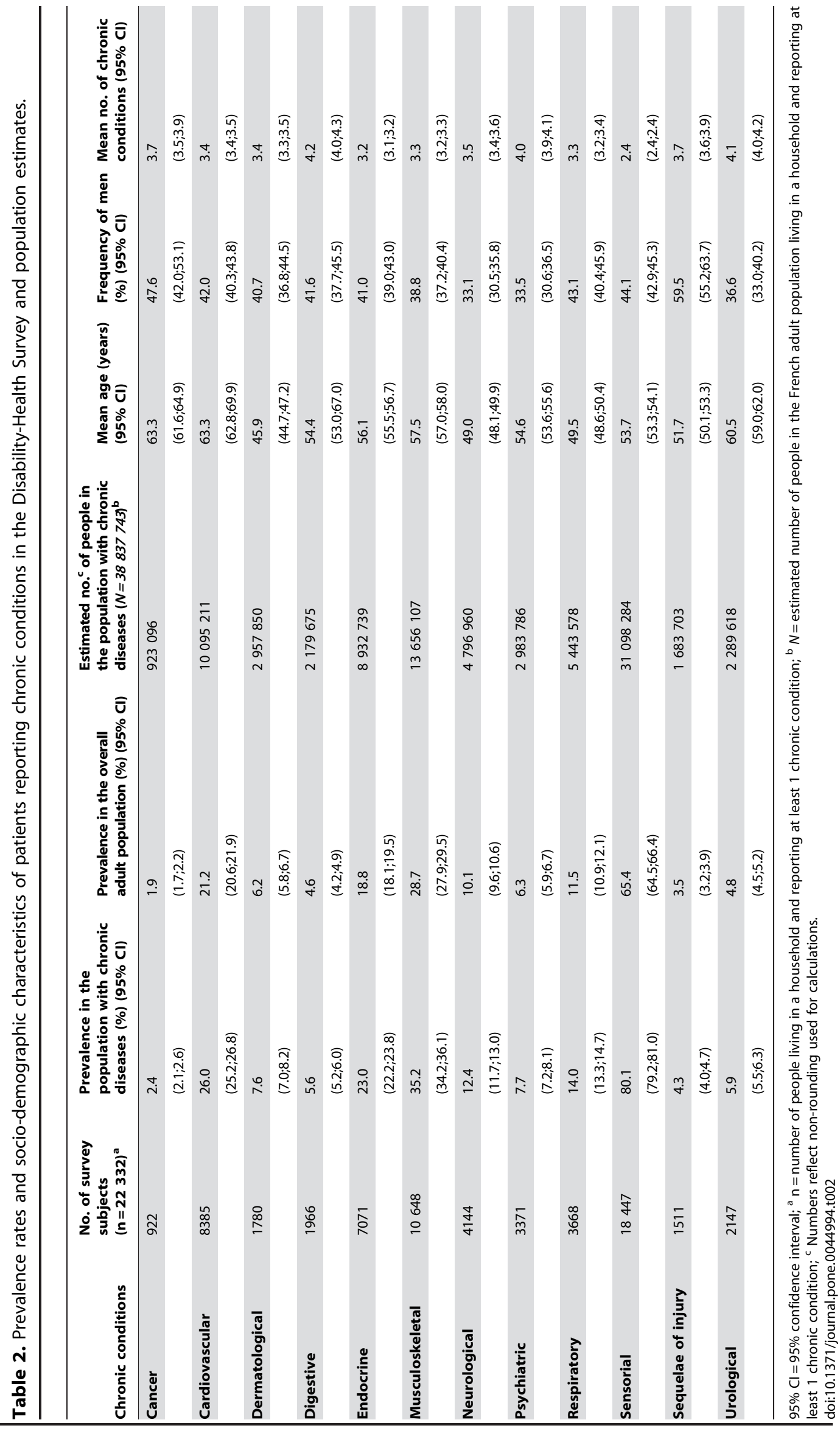


Table 3. Estimated frequency of each chronic condition in different situations of disability for people in France based on responses to the Disability-Health Survey.

\begin{tabular}{|c|c|c|c|}
\hline & Self-reported disability ${ }^{\mathrm{a}}(\%)(95 \% \mathrm{Cl})$ & Disability $^{\mathrm{b}}(\%)(95 \% \mathrm{Cl})$ & Severe disabilityc $(\%)(95 \% \mathrm{CI})$ \\
\hline Population with chronic diseases & $14.3(14.0 ; 14.6)$ & $4.6(4.4 ; 4.9)$ & $1.7(1.5 ; 1.8)$ \\
\hline Total population ${ }^{d}$ & $12.0(11.8 ; 12.3)$ & $3.9(3.7 ; 4.1)$ & $1.4(1.3 ; 1.5)$ \\
\hline Cancer & $4.9(4.3 ; 5.5)$ & $5.2(3.8 ; 6.6)$ & $5.5(3.2 ; 7.8)$ \\
\hline Cardiovascular & $43.8(42.4 ; 45.2)$ & $53.2(50.6 ; 55.8)$ & $53.3(48.9 ; 57.6)$ \\
\hline Dermatological & $8.8(8.0 ; 9.5)$ & $9.7(8.4 ; 11.1)$ & $8.2(6.7 ; 9.7)$ \\
\hline Digestive & $10.5(9.6 ; 11.3)$ & $11.5(9.9 ; 13.0)$ & $10.4(7.6 ; 13.1)$ \\
\hline Endocrine & $34.4(33.1 ; 35.8)$ & $37.3(34.8 ; 39.8)$ & $33.1(29.5 ; 36.7)$ \\
\hline Musculoskeletal & $56.3(54.9 ; 57.7)$ & $63.3(60.7 ; 65.9)$ & $50.6(46.2 ; 55.0)$ \\
\hline Neurological & $21.4(20.3 ; 22.5)$ & $32.0(29.6 ; 34.4)$ & $40.2(36.0 ; 44.4)$ \\
\hline Psychiatric & $19.8(18.8 ; 20.8)$ & $20.5(18.4 ; 22.6)$ & $22.0(18.2 ; 25.9)$ \\
\hline Respiratory & $18.5(17.4 ; 19.5)$ & $20.5(18.4 ; 22.6)$ & $22.4(18.6 ; 26.2)$ \\
\hline Sensorial & $82.8(81.7 ; 83.9)$ & $85.2(83.3 ; 87.2)$ & $82.6(79.0 ; 86.3)$ \\
\hline Sequelae of injury & $9.7(8.9 ; 10.5)$ & $10.7(8.9 ; 12.5)$ & $10.2(6.9 ; 13.5)$ \\
\hline Urological & $12.4(11.5 ; 13.3)$ & $21.4(19.1 ; 23.7)$ & $30.0(25.8 ; 34.2)$ \\
\hline
\end{tabular}

$95 \% \mathrm{Cl}=95 \%$ confidence interval;

aSelf-reported disability = positive response to the question "Do you consider that you have a disability?";

${ }^{b}$ Disability = any restriction in doing at least one activity of daily living (ADL) with or without technical aid

'Severe disability = inability to perform at least one ADL alone.

${ }^{\mathrm{d}}$ Prevalence of situations of disability in the overall adult population living in households in France.

doi:10.1371/journal.pone.0044994.t003

Table 4. Average attributable fraction ${ }^{\mathrm{a}}(\mathrm{AAF})$ estimates (\%) for each chronic disorder for disability ${ }^{\mathrm{b}}$ in France.

\begin{tabular}{|c|c|c|c|c|}
\hline Age class & $\leq 40$ & $40-65$ & $>65$ & Total Population \\
\hline Rank & $(N=143859)^{d}$ & $(N=534675)^{d}$ & $(N=1158219)^{d}$ & $(N=1836753)^{d}$ \\
\hline \multirow[t]{2}{*}{1} & Psychiatric & Musculoskeletal & Neurological & Neurological \\
\hline & 27.4 & 20.9 & 21.0 & 17.4 \\
\hline \multirow[t]{2}{*}{2} & Musculoskeletal & Endocrine & Cardiovascular & Musculoskeletal \\
\hline & 17.6 & 13.6 & 12.4 & 16.4 \\
\hline \multirow[t]{2}{*}{3} & Neurological & Neurological & Musculoskeletal & Cardiovascular \\
\hline & 11.0 & 13.3 & 10.9 & 11.1 \\
\hline \multirow[t]{2}{*}{4} & Sensorial & Psychiatric & Urological & Endocrine \\
\hline & 10.0 & 10.2 & 10.3 & 8.2 \\
\hline \multirow[t]{2}{*}{5} & Urological & Cardiovascular & Other diseases ${ }^{c}$ & Urological \\
\hline & 8.7 & 7.1 & 5.5 & 7.4 \\
\hline \multirow[t]{2}{*}{6} & Sequelae of injury & Sequelae of injury & Endocrine & Psychiatric \\
\hline & 7.6 & 6.1 & 5.2 & 5.8 \\
\hline \multirow[t]{2}{*}{7} & Cardiovascular & Sensorial & Sequelae of injury & Sensorial \\
\hline & 2.8 & 4.8 & 1.4 & 3.8 \\
\hline \multirow[t]{2}{*}{8} & Endocrine & Urological & Psychiatric & Sequelae of injury \\
\hline & 1.4 & 2.0 & 0.9 & 3.1 \\
\hline \multirow[t]{2}{*}{9} & Other diseases ${ }^{c}$ & Other diseases ${ }^{c}$ & Sensorial & Other diseases ${ }^{c}$ \\
\hline & 0.1 & 1.7 & 0.0 & 3.3 \\
\hline Sum & 86.6 & 79.7 & 67.6 & 75.3 \\
\hline
\end{tabular}

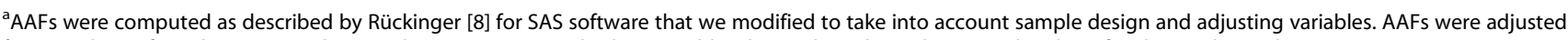
for sex, place of residence, marital status, living situation and educational level. We also adjusted on age (by class) for the total population;

${ }^{b}$ Disability = any restriction for doing at least one activity of daily living (ADL), with or without technical aid

'Other diseases = respiratory, dermatological, digestive disorders and cancer;

${ }^{\mathrm{d}} N=$ the estimated number of people in the French adult population living in household. Numbers reflect the use of a non-rounded population in calculations.

doi:10.1371/journal.pone.0044994.t004 
Table 5. Average attributable fraction ${ }^{\mathrm{a}}$ (AAF) estimates (\%) for each chronic disorder for severe disability ${ }^{\mathrm{b}}$ in France.

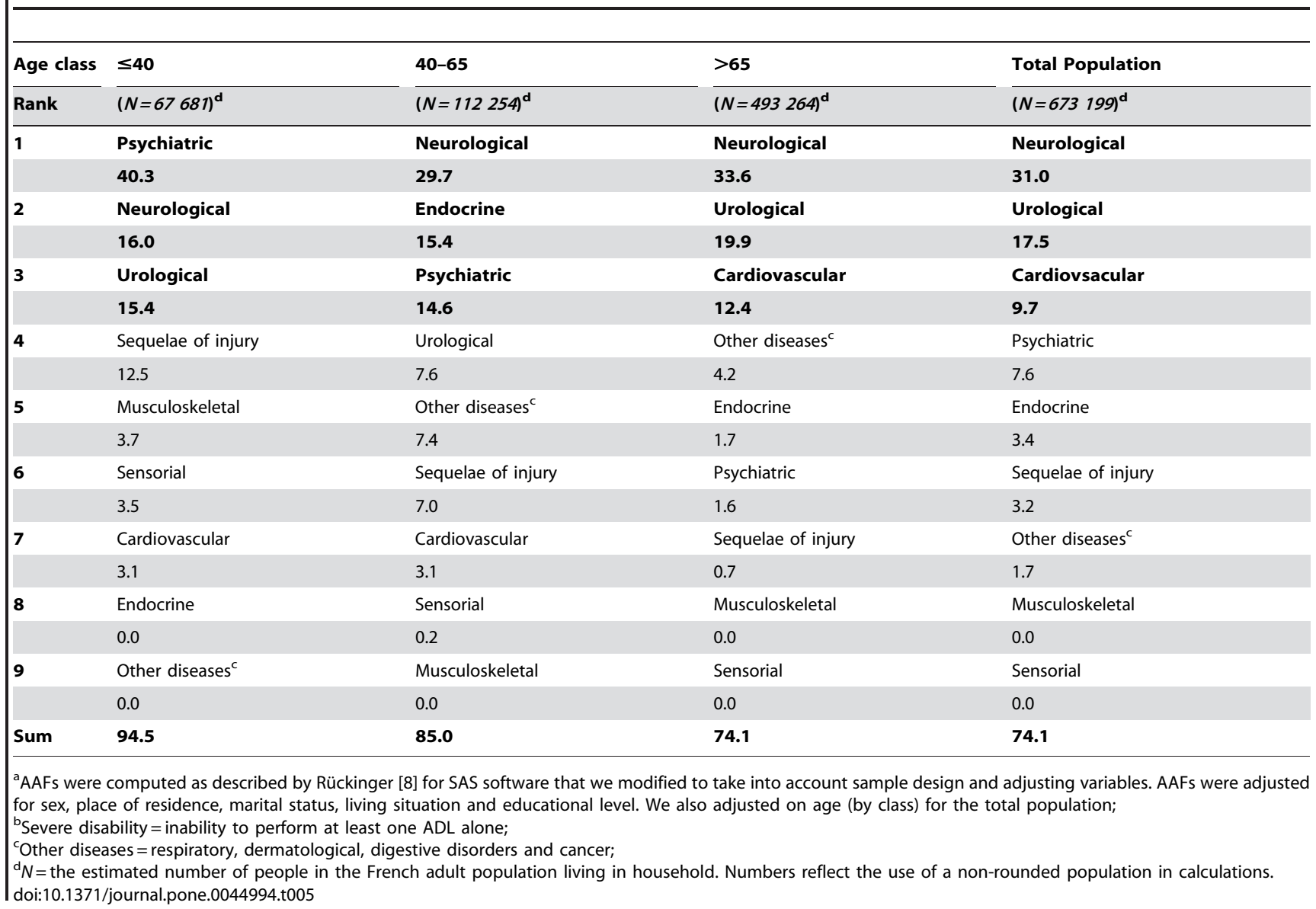

Neurological diseases had the largest impact on severe disability (AAF $31.0 \%, 210000$ people), except for people $\leq 40$ years old, for whom psychiatric disorders had the most impact $(40.3 \%$, 30000 people). The burden of musculoskeletal disorders to severe disability was insignificant.

Musculoskeletal conditions had the largest impact on selfreported disability (AAF 15.4\%, 880000 people), then sensorial and psychiatric diseases (13.2\%, 750000 people; and 9.7\%, 550000 people).

Our study revealed quantitative and qualitative differences between AAF and AF findings. Quantitatively, AAFs were lower than $\mathrm{AFs}$ for all chronic diseases, particularly for sensorial disorders $(3.8 \%$ vs. 57.3, respectively, for disability in the total population), musculoskeletal disorders (16.4\% vs. $48.5 \%)$, and cardiovascular disorders (11.1\% vs. 40.6\%). In contrast, AAFs and $\mathrm{AFs}$ for neurological diseases were close $(17.4 \%$ vs. $24.4 \%$, respectively, for disability in the total population). The sum of AAFs, which represent the part of disability attributable to chronic conditions, ranged from $67.6 \%$ to $94.5 \%$ and that of $\mathrm{AFs}$ from $117.6 \%$ to $257.9 \%$. Qualitatively, AAFs and AFs did not differ for severe disability, whatever the age class (that is the rank of chronic disorders $\mathrm{AAFs}$ and $\mathrm{AFs}$ was the same); they were mostly similar for chronic disorders. AFs were higher than $\mathrm{AAFs}$ for disability and self-reported disability for sensorial disorders but not other chronic diseases.

\section{Discussion}

Although several studies suggest that the prevalence of disability is decreasing in developed countries [11], our findings are not so optimistic. In 1999, 3.7\% of people reported at least one ADL restriction in France [21], but 3.9\% reported such restrictions in 2008-2009. The disability rate we found among people $>65$ years old in France $(12.5 \%)$ is between that for Finland $(10.2 \%)$ and England (15.3\%) [11]. The prevalence of severe disability among elderly people in France (5.3\%) is comparable to that in Canada $(5.8 \%)$ [11].

We used three indicators to identify the population reporting disability [22] - restriction in ADL and impossibility to perform ADL without help, as well as self-reported disability - which are pivotal in the International Classification of Functioning, Disability and Health (ICF) [23] and probably reflect other domains [1]. We found that the chronic conditions neurological, musculoskeletal, cardiovascular and psychiatric disorders had the largest contribution to disability in France in 2008-2009. Together, they contributed to $50.7 \%$ of disability, $48.3 \%$ of severe disability and $40.6 \%$ of self-reported disability $(63.9 \%, 65.2 \%$ and $55.3 \%$, respectively, of the part attributable to chronic conditions). These rates reflect the expected proportion of disability that could be reduced in the ideal situation where chronic disorders could be totally eliminated in France. Although this target is not realistic, a first step could be to improve prevention and treatment of these health conditions that lead to disability.

Our results can only be grossly compared with previous results from developed countries because our approach differs method- 
Table 6. Average attributable fraction ${ }^{\mathrm{a}}$ (AAF) estimates (\%) of chronic conditions for self-reported disability ${ }^{\mathrm{b}}$ in France.

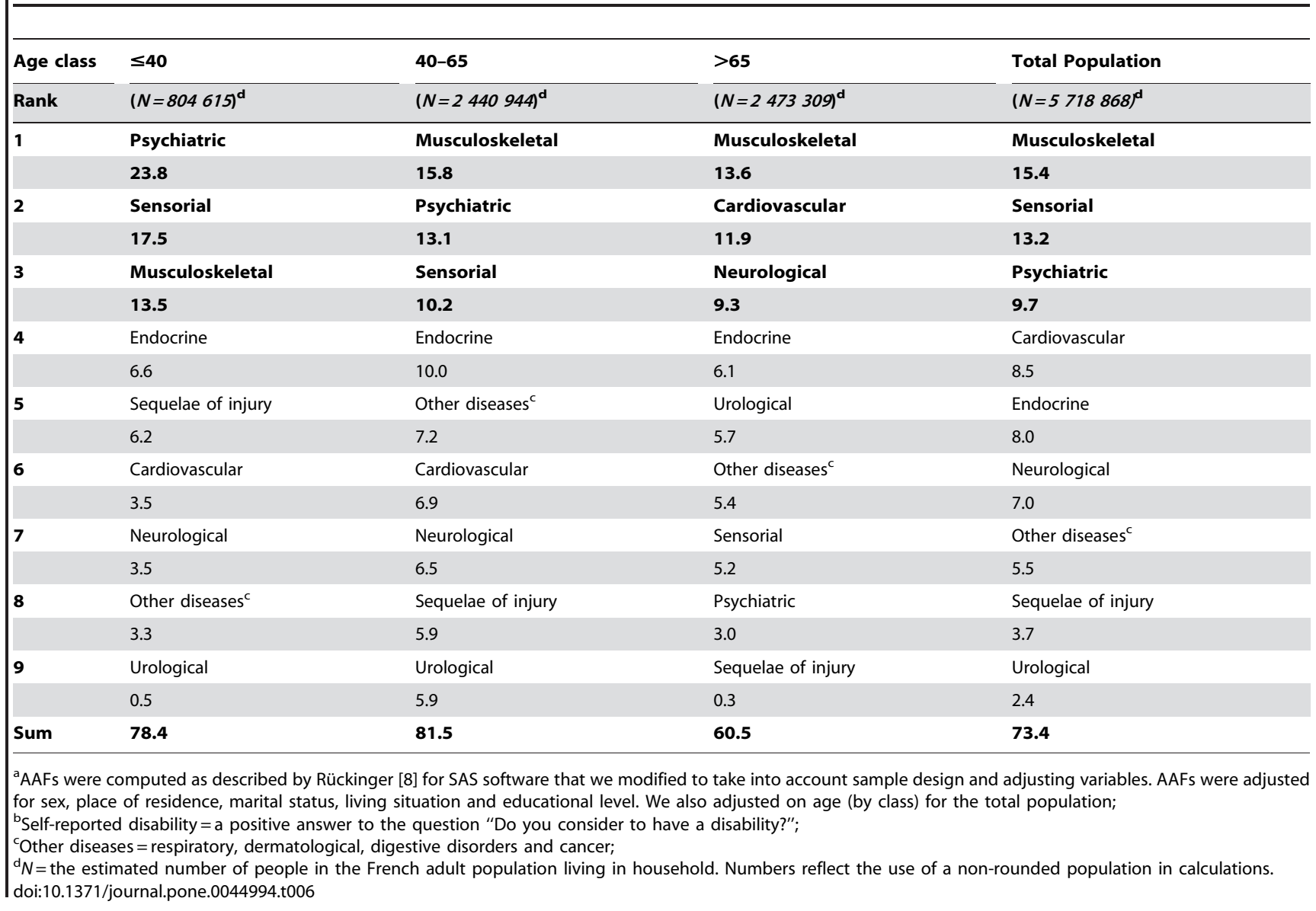

ologically from prior efforts. In a 2006 Canadian study, the most common disability-related health conditions were arthritis, back problems and hearing disorders [6], whereas in a 2001 Organization for Economic Co-operation and Development study, rheumatism was the leading cause of disability-associated conditions in elderly people in the United States [11]. In our study, the low impact of sensorial disorders and heavy burden of neurological diseases contrast with these results. These differences might be due to the lack of adjusted analyses in these studies. In fact, the high contribution of sensorial disorders in our gross analysis of disability was greatly eliminated after adjusting for age and co-morbidities, which suggests that these factors are confounders.

The respective contribution of chronic conditions differs by age, as well as definition and level of disability. Although musculoskeletal diseases had the heaviest burden in self-reported disability and the second highest impact on disability, its contribution to severe disability was almost nil. This finding suggests that people with musculoskeletal impairments often have restrictions they consider disabling (e.g., because of pain) but are rarely completely dependent in ADL. In contrast, the important contribution of neurologic conditions to severe disability in elderly people may be explained by the high level of dependence of people with neurodegenerative disorders. The high contribution of sensorial disorders to self-reported disability contrasts with the low impact on ADL restriction (severe or not). This finding shows the limitations of the Katz ADL score in that it does not include activities such as reading, sewing, cooking, or using the telephone, which involve sensorial functions. Thus, the impact of those disorders might be underestimated. We show the same findings for psychiatric disorders, which first disturb social activities that are not considered in the Katz ADL score. Finally, we highlight the significant impact of psychiatric impairments in people $\leq 40$ years old, as was suggested by recent data $[2,24,25]$, which emphasizes the need for efforts to reduce the burden of mental health problems in developed countries.

Our results cannot suggest a single priority for public health priorities in terms of which chronic condition to eliminate first. However, policymakers need information on the implications of each disability definition and the level of severity that could be relevant in disability-related programs. From our results, policymakers should focus on neurological disorders among elderly people to reduce dependence; should prevent psychiatric disorders among young people to reduce disability in this age class; and should act on musculoskeletal disorders in the overall population to improve the feeling of wellbeing, which may also reduce work absenteism in the working-age population. Examining these findings in terms of economic data would be of interest.

The main strength of this study is that our results are representative of the French household population. Thus, our data are valuable to policymakers and ensure comparison with data from other countries. Another advantage is our use of the AAF for analysis, which provides a framework for considering comorbid situations [7]. Most studies do not consider co-morbidities [5], or they use approaches not strictly valid in this situation, such as gross $\mathrm{AF}$ or adjusted odds ratios [4]. However, these methods can overestimate the potential impact of preventive strategies [8]. In fact, we found sums of unadjusted AFs higher than sums of AAFs and always exceeded $100 \%$. Such a result is not realistic and 

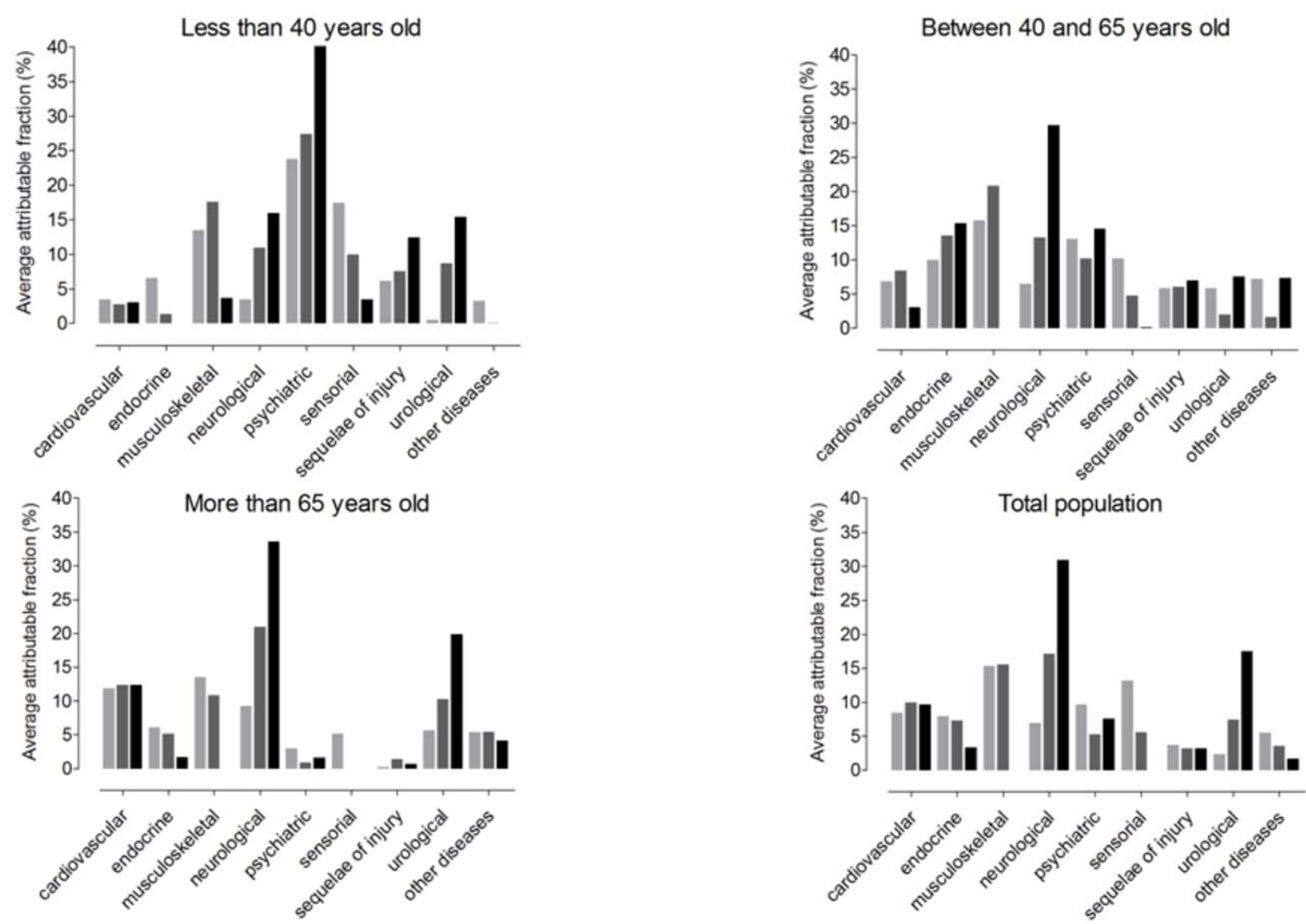

\section{Self-reported disability ${ }^{a}$}

Disability $^{\mathrm{b}}$

Severe disability ${ }^{c}$

Figure 2. Average attributable fractions of chronic conditions for different categories of disability in France and according to age. ${ }^{a}$ Self-reported disability = positive response to the question "Do you consider that you have a disability?"; ${ }^{b}$ Disability = any restriction in doing at least one activity of daily living (ADL) with or without technical aid (self-report assessment); ${ }^{c}$ Severe disability = inability to perform at least one ADL alone (self-report assessment).

doi:10.1371/journal.pone.0044994.g002

highlights the interest of AAF in analyzing co-morbidity data. On the contrary, sums of AAFs for chronic disorders never reached $100 \%$, which suggests that other factors, such as socioeconomic, environmental, and personal factors, have an impact on disability. The contribution of these factors was about $25 \%$ for the overall population and increased with age.

Our study contains some limitations. First, the Disability-Health Survey questionnaire did not collect data on several chronic disorders such as inflammatory bowel diseases or end stage chronic renal failure. However, the prevalence of these disorders is low in France $[26,27]$, so they probably contribute little to disability and their inclusion might not have changed our results greatly. We grouped diseases with $\mathrm{AFs}_{\mathrm{S}} \leq 15.0 \%$ assuming that their contribution to disability would be insignificant as compared with other conditions, which was confirmed. Because AAFs for respiratory diseases were close to $15.0 \%$, we calculated the independent $\mathrm{AAFs}$ for these diseases (data not shown) and found them to be $<2 \%$, and the AAFs for other chronic disorders did not change. Finally, we used self-reports of chronic disorders and disability. According to previous data, the accuracy of self-reporting is high for chronic disorders such as stroke [28], coronary heart disease [29] or cancer [28] but low for conditions such as arthritis [28] or non-coronary heart diseases [29]. The reliability of self-reporting in our survey is unknown. However, the prevalence rates for diseases were similar to those from other French [30] and European surveys [2]. Moreover, we used a checklist of diseases, which has been shown to yield more complete and accurate reports than estimates derived from responses to open-ended questions [31], and only diagnoses of chronic conditions by physicians were retained.

\section{Conclusions}

Neurological, musculoskeletal, cardiovascular and psychiatric disorders are the main chronic disorders contributing to disability in France. Neurological diseases have the largest impact on severe disability and in elderly people, whereas psychiatric impairments have a heavy burden in people $\leq 40$ years old. Although disability has been decreasing in developed countries, our results show that a substantial proportion of the French population feels disabled and is restricted in ADL. These findings should help health policymakers decide on priorities for health-service delivery in France and in other developed countries. These data emphasize the need to support international and national efforts to better address the main challenges with chronic diseases and disability. 


\section{Supporting Information}

Appendix S1 Method of calculation of the average attributable fraction $(\mathrm{AAF})$

(DOC)

Table S1 Sociodemographic characteristics of the population in France for each chronic condition

(DOG)

Table S2 Gross attributable fractions $\left(\mathrm{AFs}_{\mathrm{s}}\right)$ for each chronic condition estimated for the different disability categories

(DOC)

\section{References}

1. World Health Organisation (2011) World report on disability. Lancet 377: 1977.

2. World Health Organisation (2004) Prevalence for selected causes, in WHO Regions, estimates for 2004. Geneva: World Health Organisation.

3. Sousa RM, Ferri CP, Acosta D, Albanese E, Guerra M, et al. (2009) Contribution of chronic diseases to disability in elderly people in countries with low and middle incomes: a 10/66 Dementia Research Group population-based survey. Lancet 374: 1821-1830.

4. Perruccio AV, Power JD, Badley EM (2007) The relative impact of 13 chronic conditions across three different outcomes. J Epidemiol Community Health 61: 1056-1061.

5. Australian Institute of Health and Welfare Canberra (2004) Disability and its relationship to health conditions and other factors. Available http://www.aihw. gov.au/publications/dis/drhcf/drhcf.pdf. Accessed 12 August 2011.

6. Participation and Activity Limitation Survey 2006 (2006) Custom tabulation of PALS 2006 data. Statistics Canada. Available: www.statcan.gc.ca. Accessed 12 August 2011.

7. Eide GE, Gefeller O (1995) Sequential and average attributable fractions as aids in the selection of preventive strategies. J Clin Epidemiol 48: 645-655.

8. Ruckinger S, von Kries R, Toschke AM (2009) An illustration of and programs estimating attributable fractions in large scale surveys considering multiple risk factors. BMC Med Res Methodol 9: 7.

9. Katz S, Ford AB, Moskowitz RW, Jackson BA, Jaffe MW (1963) Studies of illness in the aged. The index of ADL: a standardized measure of biological and psychosocial function JAMA 185: 914-919.

10. Aijanseppa S, Notkola IL, Tijhuis M, van Staveren W, Kromhout D, et al. (2005) Physical functioning in elderly Europeans: 10 year changes in the north and south: the HALE project. J Epidemiol Community Health 59: 413-419.

11. Lafortune G, Balestat G (2007) Trends in severe disability among elderly people: assessing the evidence in 12 OECD countries and the future implications

12. World Health Organisation (2007) International Classification of Diseases (ICD). 10th revision. Geneva: World Health Organisation.

13. Levin ML (1953) The occurrence of lung cancer in man. Acta Unio Int Contra Cancrum 9: 531-541.

14. Fleiss JL (1979) Inference about population attributable risk from cross-sectional studies. Am J Epidemiol 110: 103-104.

15. Wray LA, Blaum CS (2001) Explaining the role of sex on disability: a population-based study. Gerontologist 41: 499-510.

16. Moe JO, Hagen TP (2011) Trends and variation in mild disability and functional limitations among older adults in Norway, 1986-2008. Eur J Ageing 8: 49-61.

17. Lund R, Nilsson CJ, Avlund K (2010) Can the higher risk of disability onset among older people who live alone be alleviated by strong social relations? A longitudinal study of non-disabled men and women. Age Ageing 39: 319-326.

18. Gregory PC, Szanton SL, Xue QL, Tian J, Thorpe RJ, et al. (2011) Education Predicts Incidence of Preclinical Mobility Disability in Initially High-Function-

\section{Acknowledgments}

We thank the French National Institute of Statistics and Economic Studies (INSEE), the French Head Office of Research, Studies, Evaluation and Statistics (DREES) of the Social Affairs Ministry, the Federative Research Institute on Disability (IFRH), and all the people involved in the DisabilityHealth Survey project.

\section{Author Contributions}

Conceived and designed the experiments: CP JFR PR SP. Performed the experiments: CP JFR PR SP. Analyzed the data: CP LT MD. Contributed reagents/materials/analysis tools: CP LT MD SP PR. Wrote the paper: CP.

ing Older Women. The Women's Health and Aging Study II. J Gerontol A Biol Sci Med Sci.

19. Peng X, Song S, Sullivan S, Qiu J, Wang W (2010) Ageing, the urban-rural gap and disability trends: 19 years of experience in China - 1987 to 2006. PLoS One 5: e12129.

20. SAS Institute (2008). Introduction to Survey Sampling and Analysis Procedures (Book Excerpt). Available:http://www.google.fr/url?sa $=t \& r c t=j \& q=\&$ esrc $=$ s\&source $=$ web\&cd $=1 \& \mathrm{cad}=$ rja\&ved $=0 \mathrm{CE} 8 \mathrm{QFjAA} \& \mathrm{url}=\mathrm{http} \% 3 \mathrm{~A} \% 2 \mathrm{~F} \%$ 2Fsupport.sas.com $\% 2$ Fdocumentation $\% 2 \mathrm{Fcdl} \% 2 \mathrm{Fen} \% 2$ Fstatugsurveysamp $\% 2 \mathrm{~F} 61762 \%$ 2FPDF $\% 2$ Fdefault $\% 2$ Fstatugsurveysamp.pdf \&ei $=$ Z5swUKvdJO-W0QWQsIHYDA\& usg = AFQjCNHWENrEDQIDeCsGk_Co8RrojOcffg

21. Goillot C, Mormiche P (2003) Les enquêtes Handicaps-Incapacités-Dépendance de 1998-99. Résultats détaillés. Insee Résultats SOCIETE.

22. Ravaud JF, Letourmy A, Ville I (2002) Identifying the Population with Disability: the Approach of an INSEE Survey on Daily Life and Health. Population-E 57: 529-552.

23. World Health Organisation (2001) International Classification of Functioning, Disability and Health: ICF. Geneva: WHO.

24. Gore FM, Bloem PJ, Patton GC, Ferguson J, Joseph V, et al. (2011) Global burden of disease in young people aged 10-24 years: a systematic analysis. Lancet 377: 2093-2102.

25. Kieling C, Baker-Henningham H, Belfer M, Conti G, Ertem I, et al. (2011) Child and adolescent mental health worldwide: evidence for action. Lancet 378: 1515-1525.

26. Nerich V, Monnet E, Etienne A, Louafi S, Ramee C, et al. (2006) Geographical variations of inflammatory bowel disease in France: a study based on national health insurance data. Inflamm Bowel Dis 12: 218-226.

27. Noel D, Landais P (2012) Epidemiology of chronic kidney disease. Rev Prat 62: 38-42.

28. Baumeister H, Kriston L, Bengel J, Harter M (2010) High agreement of selfreport and physician-diagnosed somatic conditions yields limited bias in examining mental-physical comorbidity. J Clin Epidemiol 63: 558-565.

29. Okura Y, Urban LH, Mahoney DW, Jacobsen SJ, Rodeheffer RJ (2004) Agreement between self-report questionnaires and medical record data was substantial for diabetes, hypertension, myocardial infarction and stroke but not for heart failure. J Clin Epidemiol 57: 1096-1103.

30. Allonier C (Juin 2007) De quoi souffre-t-on? Etat des lieux des maladies déclarées en France. Enquête décennale Santé INSEE (2002-2003). Bulletin d'information en économie de la santé 123.

31. Knight M, Stewart-Brown S, Fletcher L (2001) Estimating health needs: the impact of a checklist of conditions and quality of life measurement on health information derived from community surveys. J Public Health Med 23: 179186. 